\title{
LA CIUDAD / ELEMENTO ESPACIAL
}

\section{CITY / SPATIAL ELEMENT}

\author{
Mgter. Prof. Viviana Pértile \\ Prof. Adjunta Cátedra: Geografía Urbana y Agraria \\ Departamento de Geografía - Facultad de Humanidades -UNNE- \\ (vpertile@gmail.com). \\ (vpertile@hum.unne.edu.ar) \\ Prof. Raúl O.A.Manoiloff (+1939-2015)
}

¿Qué es la ciudad? Se puede decir que es un fenómeno de concentración permanente y de actividad en un marco espacial determinado. Concentración de población, edificios, monumentos, comercios, servicios, medios de transporte, etc.

La ciudad no es un hecho reciente; cada época histórica, a partir del Neolítico, tuvo sus hechos urbanos que hasta hoy la representan. Sin embargo en el siglo XIX se produce una transformación muy grande del hábitat, razón por la cual el crecimiento de la ciudad adquiere una dimensión hasta entonces jamás vista, extraordinaria, espectacular, que fue definida como "fenómeno urbano".

Desde 1950 a la fecha el crecimiento se acentuó aún más, tanto, que algunos geógrafos se adelantaron en la década de los 80 ' sosteniendo que la civilización del futuro será eminentemente urbana y que aproximadamente el $90 \%$ de la población vivirá en ciudades. La República Argentina cuenta ya con una población urbana superior al $90 \%$, es decir, habitando en centros de más de 2.000 habitantes.

Las razones de este crecimiento desmesurado se deben buscar en la misma humanidad que se ve impulsada a nuevas formas de civilización más urbana y más humana, a su carácter gregario, y a una ocupación cada vez más disociada de los trabajos de la tierra, como lo son la industria, el comercio, la educación, los medios de transporte, la sanidad, etc.

La máxima expresión de la ciudad es la Megalópolis.

\section{I.- Definición y características de la ciudad}

Definir la ciudad no es tema fácil ya que por ser un hecho complejo, variable e importante no hay dos ciudades iguales. No obstante, se han intentado aproximaciones para definirla a partir de ciertos criterios como el matemático o estadístico, el criterio funcional, el criterio paisajístico y el criterio histórico.

El criterio matemático tiene en cuenta la cantidad de habitantes de la agrupación; la República Argentina considera que para alcanzar la categoría de urbana, la agrupación debe tener una población mínima de 2.000 habitantes, número que también exige Francia, en cambio, Estados Unidos requiere 2.500, Canadá 1.000 y Dinamarca 250; en el otro extremo hay países que exigen 10 ó 20.000 habitantes; como se puede apreciar las diferencias son grandes, no obstante, hay que aceptar que la ciudad debe tener una población más o menos numerosa, cuando mayor sea el número más segura es su condición de hecho urbano.

Publicado en formato digital: Mgter. Prof. Viviana Pértile y Prof. Raúl O.A.Manoiloff. LA CIUDAD / ELEMENTO ESPACIAL. Revista Geográfica Digital. IGUNNE. Facultad de Humanidades. UNNE. Año 13. № 25. Enero Junio 2016. ISSN 1668-5180 Resistencia, Chaco.

En: http://hum.unne.edu.ar/revistas/geoweb/default.htm 
El criterio funcional se basa en la profesión de los habitantes urbanos, es decir, su trabajo; una especificación muy clara al respecto es la que establece que los trabajos urbanos están disociados de los de la tierra y si dentro de su población hay personas que se dedican a la agricultura $\mathrm{u}$ otra actividad primaria, éstas no deben superar el $25 \%$ de la población económicamente activa (PEA) del núcleo que se considera. Sin embargo, existen unas pocas excepciones como lo son las ciudades hortícolas de la cuenca mediterránea del Viejo Mundo y las agrociudades rusas. Los habitantes urbanos se dedican por lo general a la industria y al comercio y demás servicios.

El criterio fisonómico o paisajístico considera el aspecto de la ciudad, su paisaje, dado por un estilo, por la densidad de las construcciones, monumentos, espacios verdes, calles, etc. es decir, lo opuesto al paisaje rural.

Finalmente el criterio histórico es válido para las ciudades con un largo pasado, especie de derecho adquirido que le da la persistencia en el tiempo; de algunas ciudades, como es el caso de Roma, no se sabe como surgieron, pero fueron y son un hecho urbano indiscutible.

La mejor definición de ciudad es aquella que reúne el mayor número de criterios, como por ejemplo un número importante de habitantes, profesiones ligadas a las actividades secundarias y terciarias, con un paisaje compacto y el reconocimiento de su evolución histórica.

Max Derruau, en su Tratado de Geografía Humana (1964) define a la ciudad como una aglomeración importante, organizada para la vida colectiva (esta organización es el urbanismo) y con la mayoría de la población dedicada a las tareas no agrícolas, esta definición coincide con lo que expresáramos más arriba.

\section{I.1.- Caracteres de las ciudades actuales.}

Muchas ciudades son el resultado de la convergencia de varios procesos urbanos; allí se ubica el contenido de la geografía urbana que es estudiar las razones profundas que originan el paisaje urbano que se define en parte por negación del paisaje rural. Rastrear su evolución no es fácil como tampoco lo es hacer una tipología; sin embargo se habla de tipos urbanos, por ejemplo el de Europa Occidental, de los países desarrollados, de los subdesarrollados y otros modos de clasificarlas. Actualmente se reconocen cuatro generaciones distintas de ciudades:

1. Ciudades de primera generación: pertenecen a este tipo las ciudades más viejas (originadas en el Neolítico). Se hallan ubicadas en el Cercano Oriente, no fueron tocadas por el desarrollo industrial y crecen por efectos del movimiento natural de su población.

2. Ciudades de segunda generación: pertenecen a este tipo las ciudades nacidas en las épocas de los Grandes Imperios y en el Medioevo (ciudades amuralladas) localizadas en sitios estratégicos, preferentemente en Europa; algunas recibieron el impacto de la Revolución Industrial que las dividió en dos partes, la vieja y la nueva, un claro ejemplo de este tipo es la ciudad de Barcelona.

3. Ciudades de tercera generación: surgen en el Renacimiento y en la Edad Moderna, son el producto de la colonización europea, generalmente se ubican sobre las costas; se las encuentra en América, Asia y África. Por su situación algunas se desarrollaron vertiginosamente como es el caso de Nueva York, Buenos Aires y San Pablo. También varias de ellas fueron fundadas en el interior de los continentes, pero bien relacionadas con los puertos y a través de ellos con el

Publicado en formato digital: Mgter. Prof. Viviana Pértile y Prof. Raúl O.A.Manoiloff. LA CIUDAD / ELEMENTO ESPACIAL. Revista Geográfica Digital. IGUNNE. Facultad de Humanidades. UNNE. Año 13. № 25. Enero Junio 2016. ISSN 1668-5180 Resistencia, Chaco.

En: http://hum.unne.edu.ar/revistas/geoweb/default.htm 
exterior, podemos citar como ejemplo a Asunción, Corrientes, Santa Fe, Córdoba, Mendoza, Tucumán, etc.

4. Ciudades de cuarta generación: pertenecen a países nuevos. Se puede decir que nacieron adultas, no conocen nada de la era preindustrial. Sus planos responden a directivas muy precisas con indicaciones acerca del uso del suelo urbano, por ejemplo la plaza central, los edificios públicos en su entorno, las calles, el boulevard, los espacios verdes, el parque industrial, etc. Son ejemplos de ciudades de esta generación Reconquista, Resistencia, Formosa, Posadas, Presidencia Roque Sainz Peña, Villa Ángela y muchas otras ciudades fundadas en la Región Chaqueña, en la Pampeana y en la Patagonia.

\section{I.2.-Algunas causas que originaron las ciudades}

Beaujeu -Garnier y Chabot (op.cit.) hicieron una lista de factores que han originado y que pueden aún originarlas. Los más importantes son los siguientes: presencia de agua y posibilidad de riego (Mendoza, San Juan, San Rafael); el comercio (sitio y situación privilegiados: Buenos Aires, Rosario, Bahía Blanca); los monasterios y templos (Itatí, Lujan); el castillo; las guarniciones militares; las encrucijadas; los puertos naturales (fluviales y marítimos: Rosario, Montevideo, Santos, San Francisco, Nueva York); ciertos yacimientos mineros (carbón, petróleo y otros minerales: Comodoro Rivadavia, Río Turbio); la mentalidad de ciertos Estados modernos, con expansión política y fundaciones sistemáticas; las fundaciones industriales y las ciudades satélites.

\section{II.- El Plano de la ciudad}

\section{II.1. Situación, sitio y plano:}

La situación es la relación que tiene la ciudad con su área de influencia -hinterland, transtierra, tierra interior- que es el espacio que ordena, que organiza la ciudad. De la situación se puede decir que orienta a los hombres a elegir el sitio más adecuado dentro de determinado espacio, se refleja en las funciones de la ciudad, se materializa a través de la red de transporte y comunicación y es inseparable de los habitantes urbanos. La buena conexión de la ciudad con su área de influencia implica el desarrollo regional y el de la ciudad central.

El sitio o emplazamiento es el lugar donde está edificada físicamente la ciudad, es el terreno donde se apoya la ciudad. Pierre Lavedán, en su obra Geógraphie des Villes (1959) señala que hay cuatro causas fundamentales que llevan a elegir el sitio: alimentación y agua, defensa, religión y necesidad de relacionarse. El sitio es el producto de la elección del hombre; hay sitios favorables, como el de la ciudad de Corrientes y desfavorables como el de la ciudad de Resistencia; aunque el sitio no favorece el desarrollo de las ciudades, éstas se desarrollan en cualquier sitio cuando la situación es buena, pero los sitos buenos hacen más arquitectónicas y confortables a las ciudades.

El plano es el dibujo, diseño o trazado que representa las diferentes partes de la ciudad; es la trama determinada por las calles, los espacios edificados y los espacios libres vistos desde lo alto. La situación y el sitio se asocian para dar originalidad al plano de las ciudades. En el plano urbano se inscriben las necesidades de las funciones urbanas y se organiza la vida de todos los días. El plano más las funciones conforman la estructura urbana.

\section{II.2. Las formas típicas del plano}

Publicado en formato digital: Mgter. Prof. Viviana Pértile y Prof. Raúl O.A.Manoiloff. LA CIUDAD / ELEMENTO ESPACIAL. Revista Geográfica Digital. IGUNNE. Facultad de Humanidades. UNNE. Año 13. № 25. Enero Junio 2016. ISSN 1668-5180 Resistencia, Chaco.

En: http://hum.unne.edu.ar/revistas/geoweb/default.htm 
Existen numerosos tipos de planos, sin embargo hay formas utilizadas con más frecuencia que otras de donde resultan las llamadas formas típicas. Tal vez la más común sea el damero seguida por la radioconcéntrica.

Plano en damero: también se lo conoce como ortogonal, tablero de ajedrez, cuadrícula y cuadrillado. Es un plano difundido por los romanos al fundar las ciudades de su imperio. Su origen hay que buscarlo en la Mesopotamia asiática y en Egipto. Los españoles lo difundieron en América. Una de sus particularidades es que las calles se cortan en ángulo recto de modo que delimita espacios cuadrados y rectangulares (manzanas, entre nosotros). Entre las ventajas del plano en damero se mencionan su fácil trazado, el parcelamiento sencillo, la sectorización facilitada y la circulación ordenada; como desventaja el alargamiento de los itinerarios ya que se debe hacerlos en zig zag; la visibilidad de las esquinas, la desigual distribución de la luz solar y la posibilidad de que el viento se encauce por ciertas calles. Para resolver estos problemas, además de la construcción de ochavas, se idearon las diagonales (La Plata) y el trazado del plano a medio rumbo (Resistencia).

El plano radioconcéntrico se caracteriza por tener calles radiales y circulares. La Edad Media en Europa se menciona como la época de su expansión aunque de él también se afirma que viene de oriente. Entre las causas que los originaron se mencionan el mercado, la iglesia y el castillo. En muchas ciudades europeas las calles circulares fueron murallas que cuando se demolían se transformaban en calles, en cambio las radiales eran los ejes de circulación o mas bien de penetración al núcleo central primitivo. Entre sus ventajas se señalan el acortamiento de las distancias para llegar al centro desde la periferia y de las avenidas de circunvalación que descongestionan el tránsito y entre las desventajas la estrechez de sus calles, su irregularidad, las dificultades para establecer normas para el tránsito y la sectorización, París y Burdeos son ejemplos de este plano. Una variante del plano radioconcéntrico es el semi-radioconcéntrico que se desarrolla a partir de un obstáculo como lo pueden ser la costa del mar o la margen de un río (Amsterdan).

Otra forma de plano son: la ciudad calle, lineal o ruta, en la misma la planta urbana se desarrolla preferentemente, a lo largo de una calle por muchos kilómetros como es el caso de Eldorado en la provincia de Misiones y Volgogrado en Rusia. Brasilia.

El plano flexible adopta diferentes figuras geométricas como en Washington , Canberra y

Los planos mixtos que pertenecen a grandes y viejas ciudades, en las que cada época aportó algo que hoy se evidencia en el plano, como por ejemplo Londres, París y Berlín .

Finalmente tenemos los de disposición desordenada que presenta calles angostas y tortuosas con numerosos "cul de sac", sin líneas directrices y un mal uso del suelo urbano, pertenecen a ciudades muy antiguas de Asia, África del Norte y Europa Mediterránea.

\section{II.3. Formas de expansión de los planos.}

Toda ciudad, cuyas funciones son fundamentales para su área de influencia, crece. Dicho crecimiento se puede realizar preferentemente en tres formas: desarrollo vertical, aglutinación y extensión polinuclear o conurbación. La primera de ellas tiene lugar en el área céntrica de las grandes ciudades, debido al costo de los terrenos, la necesidad de estar en el centro y de contar con todos los servicios, en todo el mundo cada vez se construyen edificios más altos. En la aglutinación, que es la más simple, la gente construye en los bordes de la ciudad pero cerca de los accesos ruteros, donde los terrenos son más baratos y donde seguramente no se cuenta con todos los servicios. P. Geddes (1915) acuñó el término de conurbación para denominar el área urbana

Publicado en formato digital: Mgter. Prof. Viviana Pértile y Prof. Raúl O.A.Manoiloff. LA CIUDAD / ELEMENTO ESPACIAL. Revista Geográfica Digital. IGUNNE. Facultad de Humanidades. UNNE. Año 13. № 25. Enero Junio 2016. ISSN 1668-5180 Resistencia, Chaco.

En: http://hum.unne.edu.ar/revistas/geoweb/default.htm 
resultante de la coalescencia de varias ciudades o núcleos urbanos, originalmente separados, pero que el crecimiento englobó, como lo es el Gran Resistencia y el Gran Buenos Aires.

\section{4. Los suburbios}

La denominación deriva de sub-urbe, que se puede interpretar como más allá de la urbe o ciudad. Es un fenómeno propio del siglo XX. Tres factores han contribuido a su desarrollo: la multiplicación de la industria, el desarrollo de los medios de transporte y comunicación y la disociación entre los lugares de trabajo y de residencia permanente. Según la función se habla de suburbios hortícolas y eventualmente de tambos, suburbio dormitorio, industrial y de recreación; de acuerdo a su ubicación con respecto al centro de la ciudad en suburbio interior, exterior o lejano.

\section{5. Nuevas formas urbanas}

\section{II.5.a. La megalópolis}

Son la mayor expresión urbana de estos tiempos, la conurbación llevada al máximo. Este término fue establecido por Jean Gottmann al estudiar la región urbanizada del noreste de los EE.UU. comprendida entre Boston y Washington y que contiene, además de las nombradas, grandes ciudades como Nueva York, Filadelfia y Baltimore.

Es condición para ser megalópolis reunir entre el 15 y el $30 \%$ de la población de un país en menos del $5 \%$ de la superficie del mismo. Gottmann estimó la población del NE. de los EE.UU. en 38.000.000 de habitantes para 1957, cifra que ha variado poco luego de que los habitantes urbanos analizaran las difíciles condiciones de vida en las superciudades. Existen megalópolis principales y secundarias. En la República Argentina la Capital Federal y el Gran Buenos Aires, con 11.000.000 de habitantes en 1991 en 3.640 kilómetros cuadrados, también constituye un claro ejemplo de megalópolis.- Algunos urbanistas incluyen en la megalópolis el área comprendida entre La Plata y Santa Fe. La Megalópolis es un hecho reciente, complejo, grande, fluído y distinto según los países.

\section{II.5.b. Las ciudades satélites}

Fueron pensadas para descentralizar las grandes ciudades; las idearon los rusos que le pusieron el nombre de "Sputnik" a semejanza de los satélites que circunvalan la tierra; también la adoptaron los ingleses los que la llamaron "New Town". Estas ciudades cumplen con algunas funciones como ser que los habitantes de las ciudades satélites vayan a trabajar a la ciudad centro; que la ciudad centro les asegure un cierto número de servicios (especialmente culturales) a su vez las ciudades satélites reciben población de la ciudad centro con fines recreativos y que la ciudad centro es por lo menos diez veces mayor que las satélites. Se estima que las ciudades satélites no deben tener más de 300.000 habitantes, de lo contrario, comienzan a reproducir los problemas de las grandes ciudades. Otra forma de descentralizar las grandes ciudades es creando barrios equipados para satisfacer las necesidades básicas de la población.

\section{Las funciones urbanas}

La definición de ciudad señala que es una forma de agrupamiento permanente y de actividad en un marco espacial determinado. En cuanto a las actividades de los habitantes urbanos hay que hacer una distinción entre función y servicio. Las funciones son actividades que realizan

Publicado en formato digital: Mgter. Prof. Viviana Pértile y Prof. Raúl O.A.Manoiloff. LA CIUDAD / ELEMENTO ESPACIAL. Revista Geográfica Digital. IGUNNE. Facultad de Humanidades. UNNE. Año 13. N N 25 . Enero Junio 2016. ISSN 1668-5180 Resistencia, Chaco.

En: http://hum.unne.edu.ar/revistas/geoweb/default.htm 
los habitantes urbanos volcadas hacia el exterior, se sobreentiende que también se hacen para los pobladores de la ciudad, por su parte los servicios son actividades que de preferencia se hacen para satisfacer las necesidades de la ciudad /alumbrado, recolección de residuos, barrido, limpieza, etc.). Los hombres se agrupan y forman ciudades para ejercer ciertas formas de actividad; esas actividades son la razón de ser de las ciudades; las ciudades viven y se desarrollan por ellas y prácticamente no hay ciudades sin funciones. Muchas veces la función, cuando se destaca, se traduce en el aspecto exterior de la ciudad, por ejemplo la portuaria, la de ciertas industrias, la universitaria, la turística, la religiosa, etc.

El problema más difícil de resolver es cómo se determinan las funciones urbanas. Es un trabajo que supone investigación, encuestas, análisis, y comparaciones. El trabajo de investigación llevado a cabo por el Instituto de Geografía de la Facultad de Humanidades de la UNNE y publicado en la Revista Geográfica Números 1, 2 y 3 es un ejemplo de determinación de las funciones urbanas para el caso de Resistencia.

Beaujeu-Garnier y Chabot en su Tratado de Geografía Urbana hacen una clasificación muy completa de las funciones urbanas, cuya síntesis es la siguiente:

a.- Función militar: actualmente muy disminuida pero muy importante por haber sido el origen de muchas ciudades. La República Argentina tiene buenos ejemplos, como el de Paso de los Libres y Puerto Belgrano.

b.- Función comercial: está presente en todas las ciudades y a menudo es la principal de las funciones, como en Resistencia.

c.- Función industrial: llamada por su importancia, madre de ciudades, eje. San Nicolás.

d.- Función cultural: se subdivide en función religiosa, las ciudades universitarias, los centros literarios y artísticos, las ciudades museos y las ciudades de festivales y de congresos.

e.- Función de acogida: corresponde a las ciudades de hospitales y de sanatorios; las ciudades de esparcimiento, veraneo y turismo y las ciudades de retiro, como Mar del Plata y San Carlos de Bariloche.

f.- Función administrativa y política: comprende la administración y está representada por las capitales a nivel de provincia o país y también las capitales mundiales o supercapitales., La Plata, Buenos Aires, París, Nueva York.

\section{IV.. La estructura urbana.}

Hasta hace poco la estructura de una ciudad se consideraba como cosa física, como algo estático y rígido. Si bien se tenía en cuenta el crecimiento y el cambio, estos no eran las ideas centrales. La ciudad debe ser considerada como un sistema espacial en continuo cambio, en movimiento. La estructura entonces se presenta como un sistema espacial. Los elementos que componen la estructura o sistema necesitan una ubicación en el espacio urbano que le permita relacionarse con otros elementos.

El conjunto de actividades urbanas y las relaciones que mantienen entre sí constituye el sistema urbano. Este sistema está organizado sobre subsistemas de componentes, de manera que cualquier cambio en los subconjuntos implica cambios en el sistema.

Todo sistema urbano está basado en seis principios fundamentales:

1.- Las actividades de la ciudad se vinculan con otras actividades que están fuera de ellas. La estructura urbana no debe considerarse aisladamente, sino junto a su región y con otras ciudades de la armadura nacional y mundial.

2.- La estructura urbana comprende múltiples elementos cuyo ritmo de evolución es variable.

3.- El sistema o la estructura urbana es básicamente dinámica.

4.- Los elementos físicos de la estructura son el receptáculo de los elementos socioeconómicos, lo que permite el desarrollo de las actividades y a la vez son el resultado de esas actividades.

Publicado en formato digital: Mgter. Prof. Viviana Pértile y Prof. Raúl O.A.Manoiloff. LA CIUDAD / ELEMENTO ESPACIAL. Revista Geográfica Digital. IGUNNE. Facultad de Humanidades. UNNE. Año 13. № 25. Enero Junio 2016. ISSN 1668-5180 Resistencia, Chaco.

En: http://hum.unne.edu.ar/revistas/geoweb/default.htm 
5.- Los elementos físicos son de mayor perdurabilidad que los elementos socioeconómicos.

6.- Las tensiones y desequilibrios en la ciudad se deben al desfase entre los elementos físicos (perdurables) y los económicos (no perdurables y de evolución más rápida).

\section{IV.1. Elementos de la estructura}

Los elementos de la estructura se pueden clasificar en físicos, socioeconómicos y extraurbanos.

Elementos físicos: a su vez se dividen en cuatro subgrupos: redes de transportes, espacios libres, espacios edificados y los caracteres del sitio.

Las redes de transporte están formadas por la calle (origen, función, estructura), las vías férreas y los canales, si los hubiere. Los espacios libres comprenden las plazas (públicas o de reunión, las monumentales, de tráfico y de comercio), los jardines y los parques. Los espacios edificados y sus funciones pueden ser residenciales (de lujo, obreros o de emergencia), comerciales (al por mayor y menor), industriales, de servicios y las construcciones públicas . Los caracteres del sitio responden básicamente a la topografía (llano, ondulado, escarpado) y la posición geográfica (litoral, interior, de valle, de piedemonte, etc.).

Elementos socioeconómicos: dentro de este capítulo se ubica la estructura propietaria (pública, privada), la población y sus caracteres (distribución, estructura, movimiento natural y migratorio), el aspecto cultural y las actividades productoras de bienes y servicios.

Los elementos extraurbanos, comprenden el área de influencia y sus características, la región, el país y su armadura urbana.

\section{2- Los barrios urbanos.}

Toda ciudad, grande o pequeña, se divide en partes que comúnmente se denominan barrios, que tienen cada uno, sus particularidades desde el punto de vista físico, humano y económico.

Los barrios más comunes y fáciles de destacar son los siguientes:

1 Barrio de comercio y negocios

2 Barrio Industrial

3 Barrio residencial

4 Barrio universitario

5 Barrio político administrativo

6 Barrio de la estación (ferroviaria y de ómnibus)

7 Barrio residenciales de emergencia (villas miserias, chabolas, barriadas, favelas).

En la actualidad, prácticamente todas las ciudades del mundo tratan de organizar el uso del suelo urbano, pero además de la zonificación, las distinciones barriales pueden obedecer a razones históricas, étnicas, religiosas y económicas.

\section{V.- Demografía urbana}

El análisis de la población de una ciudad comprende varios aspectos, uno de ello es la densidad. Calcular la densidad de la población de una ciudad es una tarea bastante complicada, a menos que la misma esté perfectamente delimitada, pero como en la realidad no es así hay que

Publicado en formato digital: Mgter. Prof. Viviana Pértile y Prof. Raúl O.A.Manoiloff. LA CIUDAD / ELEMENTO ESPACIAL. Revista Geográfica Digital. IGUNNE. Facultad de Humanidades. UNNE. Año 13. № 25. Enero Junio 2016. ISSN 1668-5180 Resistencia, Chaco.

En: http://hum.unne.edu.ar/revistas/geoweb/default.htm 
buscar la forma más adecuada para hacerlo, por ejemplo, calcularla por "manzana", por calles, por superficie cubierta, por vivienda, por barrio. En nuestro país se la podría obtener por fracciones, radios y segmentos censales.

Otro aspecto que podemos analizar es la composición por edad y sexo de la población urbana, ello nos permitirá saber si la población es joven o vieja y la proporción de los sexos o índice de masculinidad. Las pirámides de población revelan fácilmente estos temas.

El origen de la población es otro tema de la estructura de la población urbana. La ciudad fue siempre un poderoso centro de atracción de inmigrantes, por eso se dice que es un crisol de razas ya que se puebla con personas provenientes de numerosos lugares; los individuos de cada raza, religión o nacionalidad tienden a reunirse y formar sus propios barrios en las ciudades.

La composición profesional de la población de la ciudad demuestra que hay variedad de profesiones. Predominan los secundarios (trabajadores de la industria) y los terciarios (empleados en los servicios).

Los que trabajan en tareas rurales son los menos representados, salvo algunas excepciones como ya lo hemos dicho para Valencia.

La ciudad presenta, también, un movimiento natural propio que estará de acuerdo con la edad de la población y con el nivel sociocultural, por eso en la ciudad por lo general la natalidad es más baja que en las áreas rurales, la mortalidad puede ser mayor o menor según la composición etaria, la mortalidad infantil es mas baja y el crecimiento vegetativo semejante al de las áreas rurales.

El movimiento migratorio de las ciudades está fundamentado en el poder de atracción de la ciudad, tomada globalmente. Cuando las áreas rurales se saturan de población, los excedentes se trasladan a las ciudades que, aun cuando no están preparadas para recibir dichos excedentes terminan por incorporarlos a su estructura.

\section{V.I.- Algunos caracteres demográficos de América Latina}

Si bien las fuentes de información son desiguales como desigual es el grado de urbanización de América Latina, se han obtenido algunas características comunes para el conjunto de países que la integran:

1.- En las grandes ciudades la tasa de fecundidad es débil.

2.- El índice de masculinidad es menor que en las áreas rurales.

3.- La nupcialidad es mayor (todavía).

4.- La proporción de jóvenes y adultos es elevada.

5.- La tasa de analfabetos es sensiblemente menor.

6.- La tasa de mortalidad general no presenta diferencias, pero la mortalidad infantil es menor en las ciudades.

7.- El rápido crecimiento de las ciudades, en particular de las más grandes se explica esencialmente por las migraciones campo-ciudad.

8.- El crecimiento natural es semejante en áreas urbanas y rurales.

9.- La población rural que se instala en las ciudades es en su mayoría de sexo femenino de entre 15 y 39 años.

10.- De las migraciones internacionales que contribuyeron al crecimiento de las ciudades, predominó el sexo masculino.

11.- El número de activos masculinos y de obreros industriales es semejante a la de los países más evolucionados, el de los servicios, es mayor y en el de comercio, es menor.

Publicado en formato digital: Mgter. Prof. Viviana Pértile y Prof. Raúl O.A.Manoiloff. LA CIUDAD / ELEMENTO ESPACIAL. Revista Geográfica Digital. IGUNNE. Facultad de Humanidades. UNNE. Año 13. № 25. Enero Junio 2016. ISSN 1668-5180 Resistencia, Chaco.

En: http://hum.unne.edu.ar/revistas/geoweb/default.htm 
Revista Geográfica Digital. IGUNNE. Facultad de Humanidades. UNNE. Año 13. № 25.

Enero - Junio 2016. ISSN 1668-5180 Resistencia, Chaco

\section{VI.- La ciudad y la región}

La ciudad es un hecho destacable en el espacio, pero su comportamiento no es semejante al de una isla. La ciudad existe por la región, la región a la vez, se organiza, vive y se desarrolla a través de la ciudad, por lo tanto es un sistema integrado e indivisible. Las relaciones entre la ciudad y la región no son permanentes, es decir que varían con el tiempo; el punto de partida entre la ciudad y la región es el campo que produce, la ciudad que elabora, conserva o mejora y devuelve los productos transformados. En la actualidad el desarrollo de los medios de transporte y comunicación desnaturaliza la relación campo ciudad, sin embargo, las relaciones persisten y a partir de ellas se elaboran las políticas para el desarrollo.

El concepto de región es muy usado pues todo el mundo habla de ella aunque con diferente significación. Las primeras regiones concebidas fueron las físicas o naturales, más adelante aparecieron la región geográfica, la histórica, la económica y muchas otras. El geógrafo francés André Cholley clasifica a las regiones de la siguiente manera: son dominios cuando se refiere a los elementos naturales, son medios cuando a los dominios se le agrega la vegetación natural y son regiones cuando aparece el hombre con poder transformador, es decir que no concibe la región sin la presencia humana racional; muchos franceses comparten su criterio.

François Perrou, también francés, reconoce tres tipos de regiones: 1) la región homogénea, cuando uno de los elementos (físico o humano) es constante; 2) la región programa o plan, es aquella delimitada por un problema la que se le quiere dar solución, por ejemplo la región del mal de chagas, del vinal, de las moscas de la fruta, del picudo del algodonero, etc. 3) la región funcional, nodal o polar, es la región que se organiza a partir de un centro que es la ciudad. Allí la ciudad es una concentración de energía que irradia hacia el exterior, es el "cerebro" que regula la vida de un espacio. La región urbana es entonces, la ciudad y su área de influencia. La ciudad, a partir de cada función, tiene numerosas áreas de influencia, cuyos límites se fijan según la escala y los objetivos.

\section{VII.- Distribución espacial de las ciudades}

El simple análisis cartográfico de la armadura urbana de una provincia, región o país sugiere una serie de interrogantes como los siguientes: ¿las ciudades y su tamaño se distribuyen al azar? ¿existen leyes que determinan la distribución? ¿las distancias entre las ciudades son fruto de la casualidad? ¿qué factores influyen? ¿por qué hay ciudades grandes y pequeñas? ¿las ciudades grandes se ubican en cualquier parte? ¿hay ubicaciones preferenciales que obedecen a ciertas normas? ¿por qué en el crecimiento de las ciudades hay notables diferencias? ¿hay teorías que hablan del tamaño, de las distancias y de la jerarquía de las ciudades?

Efectivamente, hay teorías que se refieren al tamaño, naturaleza y a la distribución de las ciudades. Una de las primeras fue la de Walter Christaller. Este economista realizó en 1933 un estudio de las ciudades de la región alemana de Baviera; para él las ciudades se comportan como centros de mercado.

Christaller llega a una serie de conclusiones como ser que hay cierto orden en la distribución de pueblos y ciudades. La distribución espacial refleja cierta adaptación al factor distancia (distancia-costo, distancia-tiempo).

Otro factor que incide en la distribución de los centros urbanos es la accesibilidad, las de mayor abordabilidad son las que más crecen; este factor se mide por el número de carreteras que convergen al lugar y la calidad de las mismas. Las ciudades que tienen mejores condiciones de situación se desarrollan más rápidamente, aun cuando su sitio no sea el mejor.

Finalmente Walter Christaller establece una jerarquía de mercados a los que reúne en: 1.- Mercados locales: ofrecen bienes y servicios comunes.

2.- Mercados regionales: ofrecen bienes y servicios que se compran con menos frecuencia.

Publicado en formato digital: Mgter. Prof. Viviana Pértile y Prof. Raúl O.A.Manoiloff. LA CIUDAD / ELEMENTO ESPACIAL. Revista Geográfica Digital. IGUNNE. Facultad de Humanidades. UNNE. Año 13. N N 25 . Enero Junio 2016. ISSN 1668-5180 Resistencia, Chaco.

En: http://hum.unne.edu.ar/revistas/geoweb/default.htm 
3.- Mercados nacionales: ofrecen bienes y servicios muy raros que se compran ocasionalmente.

El aporte de Christaller tuvo el mérito de promover estudios sistemáticos acerca de la distribución de las ciudades, aun cuando el desarrollo de los medios de transporte y comunicación han desnaturalizado el valor de algunas de sus premisas como por ejemplo el factor distancia , el tema de las ciudades adquiere cada vez más espacio en la disciplina geográfica.

Publicado en formato digital: Mgter. Prof. Viviana Pértile y Prof. Raúl O.A.Manoiloff. LA CIUDAD / ELEMENTO ESPACIAL. Revista Geográfica Digital. IGUNNE. Facultad de Humanidades. UNNE. Año 13. № 25. Enero Junio 2016. ISSN 1668-5180 Resistencia, Chaco.

En: http://hum.unne.edu.ar/revistas/geoweb/default.htm 


\section{BIBLIOGRAFÍA}

- Beaujeu Garnier, J.y Chabot, Georges (1975): Tratado de Geografía Urbana. Ed. Vicens Vives, Barcelona. Carter, Harold (1974): El estudio de la Geografía Urbana. Ed. Instituto de Estudios de Administración Local. Madrid.

- Derruau, Max (1967):Tratado de Geografía Humana. Ed. V. Vives. Barcelona.

- Dickinson, Robert (1961: Ciudad, Región y Regionalismo. Ed. Omega. Barcelona.

- Gutiérrez Puebla, Javier (1984): La ciudad y la organización regional. Ed. Cincel. Madrid.

- Hernando Rica, Agustín (1992): Hacia un mundo de ciudades. El proceso de urbanización. Ed. Cincel. Madrid.

- Instituto de Geografía (1972, 1973 y 1974): Geográficas 1, 2 y 3. Resistencia, Chaco.

- Johnson, James (1980): Geografía Urbana. Ed. Oikos. Barcelona.

- Jones, Emrys (1973): Pueblos y ciudades. Ed. Eudeba. Bs.As.

- Lavedán, Pierre (1959): Geógraphie de Villes. Ed. Gallimard. París.

- Randle, Patricio (1977): La ciudad pampeana. Ed. Oikos. Bs.As.

- Souza, María A. de (1994): A identidade da metrópole. A verticalizaçao em Sao Paulo. Ed. HUCITEC - EDUSP. San Pablo.

- Zamorano, Mariano (1977): Geografía urbana. Formas económicas y dinámicas de las ciudades . Colección Geográfica № 6 . Buenos Aires

Publicado en formato digital: Mgter. Prof. Viviana Pértile y Prof. Raúl O.A.Manoiloff. LA CIUDAD / ELEMENTO ESPACIAL. Revista Geográfica Digital. IGUNNE. Facultad de Humanidades. UNNE. Año 13. N N 25. Enero Junio 2016. ISSN 1668-5180 Resistencia, Chaco.

En: http://hum.unne.edu.ar/revistas/geoweb/default.htm 